\title{
TOWARDS INSAR EVERYWHERE, ALL THE TIME, WITH SENTINEL-1
}

\author{
Zhenhong Li $^{\mathrm{a}, *}$, Tim Wright ${ }^{\mathrm{b}}$, Andrew Hooper ${ }^{\mathrm{b}}$, Paola Crippa ${ }^{\mathrm{a}}$, Pablo Gonzalez ${ }^{\mathrm{b}}$, Richard Walters ${ }^{\mathrm{c}}$, John Elliott ${ }^{\mathrm{d}}$, Susanna \\ Ebmeier $^{\mathrm{e}}$, Emma Hatton ${ }^{\mathrm{b}}$, Barry Parsons ${ }^{\mathrm{d}}$ \\ ${ }^{a}$ COMET, School of Civil Engineering and Geosciences, Newcastle University, Newcastle upon Tyne, NE1 7RU UK - \\ (Zhenhong.Li, Paola.Crippa)@newcastle.ac.uk \\ ${ }^{\mathrm{b}}$ COMET, School of Earth and Environment, University of Leeds, Leeds LS2 9JT, UK - (t.j.wright, a.hooper, P.J.Gonzalez, \\ E.L.Hatton)@leeds.ac.uk \\ ${ }^{\mathrm{c}}$ COMET, Department of Earth Sciences, Durham University, Durham DH1 3LE, UK - richard.walters@durham.ac.uk \\ ${ }^{\mathrm{d}}$ COMET, Department of Earth Sciences, University of Oxford, Oxford OX1 3AN, UK - (john.elliott, \\ barry.parsons)@earth.ox.ac.uk \\ ${ }^{\mathrm{e}}$ COMET, School of Earth Sciences, University of Bristol, Bristol BS8 1RJ, UK - sk.ebmeier@bristol.ac.uk
}

\section{Commission VI, WG VI/4}

KEY WORDS: Sentinel-1, TOPS, InSAR, ECMWF, Tectonics, Volcanoes

\begin{abstract}
:
Sentinel-1A was launched in April 2014, and has been collecting data routinely over more than one year. Sentinel-1B is set for launch on 22 April 2016. The Sentinel-1 constellation has several advantages over previous radar missions for InSAR applications: (1) Data are being acquired systematically for tectonic and volcanic areas, (2) Images cover a wide footprint, $250 \mathrm{~km}$ from near to far range in Interferometric Wide Swath (TOPS) mode, (3) Small perpendicular and temporal baselines greatly improve interferometric coherence at C-band, (4) Data are freely available to all users, (5) The mission is planned to be operational for 20 years, with 1C and 1D planned for future launches. These features enable us to map geological processes occurring in any place at anytime using InSAR.
\end{abstract}

We will review progress within COMET towards our ultimate goal of building a fully-automated processing system that provides deformation results and derived products to the wide InSAR and Geophysics communities. In addition to high-resolution-ECMWFbased atmospheric correction model, we will show results of a systematic analysis of interferometric coherence in tectonic and volcanic areas, and discuss the future goals and timeline for the COMET InSAR automated processing system.

\section{INTRODUCTION}

Sentinel-1A was launched in April 2014 and its data have been widely exploited for a range of applications including the monitoring of land surface movements due to geohazards, mapping of ice-sheet, surveillance of marine environments (oilspill and ship detection), and mapping for agriculture, forest, water and soil management. Its identical twin, Sentinel-1B is set for launch on 22 April 2016. Once Sentinel-1B is in orbit, the Sentinel-1 mission will be able to image every place on the Earth every six days, and even more frequently in some places. The Sentinel-1 mission is planned to provide systematic observations of tectonic and volcanic areas at global level. Its all-weather, dayor-night capability, together the wide swath of $250 \mathrm{~km}$ in the Interferometric Wide Swath mode, make itself particularly suitable for monitoring geohazards such as earthquakes, volcanoes and landslides. The shorter repeat time, more regular acquisitions and tighter orbital control for Sentinel-1 radically improve the coherence of interferograms relative to previous ERS-1/2 and Envisat missions and allow interferometric processing and time series analysis to be automated.

\section{COMET INSAR AUTOMATIC PROCESSING CHAIN}

Funded by the UK Natural Environmental Research Council (NERC) through the Looking Inside the Continents (LiCS) project, COMET scientists have been developing a fully automated, state-of-the-art InSAR processing chain for Sentinel-1 data. The COMET InSAR automated system has its origin in the years 2014-2015 when most of the work was related with the capacity building stage, adaptation of existing technology to the TOPS processing particularities of the Sentinel-1 and applying it on-the-fly to on-going geophysical events, such as the 2014-2015 Fogo volcano eruption (Gonzalez et al., 2015), or the April 2015 Nepal earthquake (Elliott et al., 2016).

Sentinel-1 mission by default acquires SAR data in burst-mode. Each Terrain Observation with Progressive Scans SAR (TOPSAR) image is composed of three or five subswaths, for Interferometric Wide and Extended Wide Swath mode, respectively. Every burst acquired by the constellation has to be precisely synchronized, 5 milliseconds at most, to be valid for interferometric processing. A single acquisition of Sentinel-1 data can be up to $25 \mathrm{sec}$ long. However, each burst ( $2.7 \mathrm{sec}$ long) covers the same geographical area, with enough spatial overlap to ensure data continuity. We exploit the synchronization of the bursts to internally organize the COMET InSAR processing chain. We predefine frames, each frame contains a variable, but fixed, number of bursts. A frame concept for processing helps to obtain a robust and consistent processing unit level, which has benefits for interferometric processing, as explained below.

In early 2016, the LiCS project team within COMET started to build the COMET InSAR automated system on the Facility for

* Corresponding author 
Climate and Environmental Monitoring from Space (CEMS) at Harwell with around $425 \mathrm{~TB}$ of archived products and 100 processing cores. CEMS has the advantage that it has a direct data pipeline to the main Sentinel-1 Processing and Archiving Facility, based nearby at Farnborough, and has already established methods and protocols for serving large data volumes to the community.

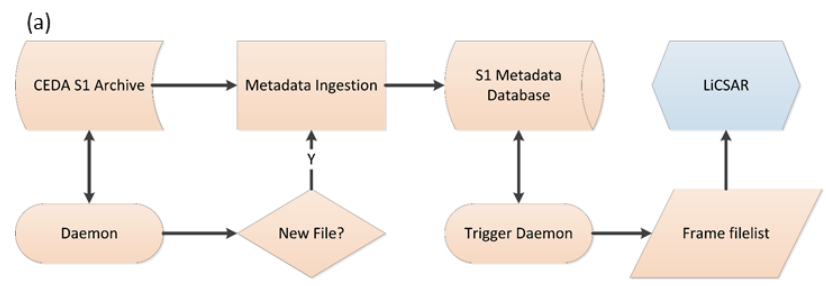

(b)

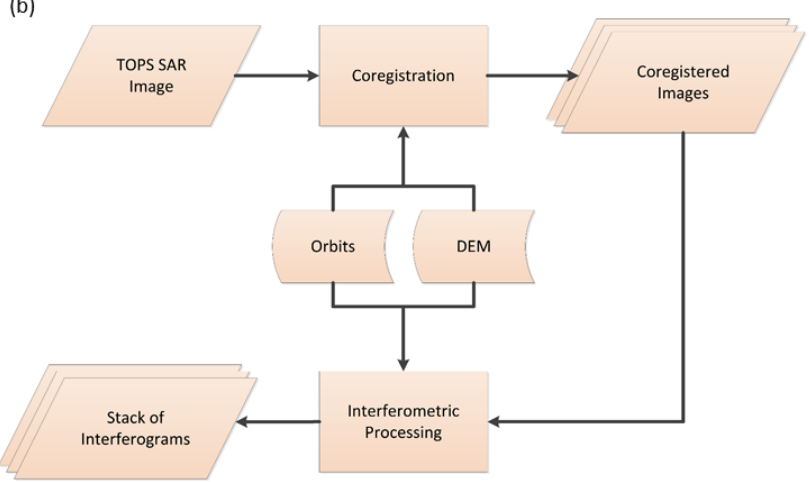

(c)

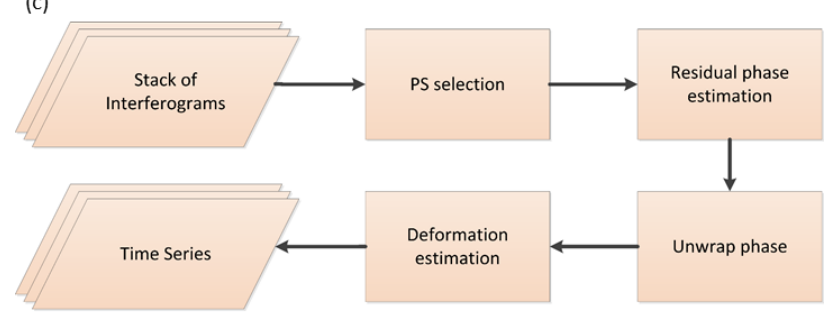

Figure 2. COMET InSAR automated system workflow. Panel (a) shows the database ingestion component and InSAR triggering mechanism. LiCSAR, the interferometric processing chain is shown in panel (b). Panel (c) depicts the time series estimation steps.

Automatic data processing relies on a database of Sentinel-1 metadata designed to contain product level information (Figure 1a), such as data acquisition time and burst-level information including geographic coverage. In order to ensure consistent processing, the burst information is used to construct defined frames for processing with GAMMA software (www.gamma-rs.ch).

The processing chain is triggered when a daemon, an automatic program running in the background on our servers, detects new Sentinel-1 data in the archive at the Centre for Environmental Data Analysis (CEDA, www.ceda.ac.uk). Then, the metadata of the new product is ingested into the database. A second daemon monitors the database for newly ingested information at specified time intervals. We spatially filter the information to match our specified frames, and a list of the Level 1, Single Look Complex (SLC) zipfiles is sent for interferometric processing (Figure 2b).
New scenes are concatenated, mosaicked and cropped to fit within the frames, generating single SLC for each new date. The next step is to obtain precisely co-registered SLCs (Figure 1b). To align the SLCs, we follow the procedures outline in González et al. (2015). We briefly describe it here. Terrain Observation with Progressive Scans (TOPS) acquisition mode implies an electronic scanning in the azimuth direction (satellite along-track direction). The sweeping process induces a variable Doppler, which affects the interferometric phase. If not accurately accounted for, it can cause phase discontinuities at the burst boundaries. The precise co-registration of the SLC images required for TOPS mode processing is performed in two stages, during which constant azimuth and range offsets are estimated and iteratively refined. First, the slave image is geometrically co-registered to the master scene using precise orbit information and a Digital Elevation Model (DEM). Optionally, offsets between the master and resampled slave images are estimated using the cross-correlation. Finally, the offsets are iteratively re-estimated using the Doppler variation in the region of burst overlap.

Once, the new SLCs are coregistered, we construct, by default, the three shortest temporal baseline interferograms. Using a two-pass interferometric approach, we compute the differential interferograms. Topographic phase is simulated using the precise orbits and an external DEM. For regions, with -58 degrees and 60 degrees in latitude we use the newly released 1-arcsec Shuttle Radar Topography Mission (SRTM) 30m DEM (Farr et al., 2007). Differential interferograms, for each frame, are formed at a default 4 looks in azimuth and 20 looks in range to obtain a pixel size of $\sim 100 \times 100 \mathrm{~m}^{2}$. A quality assessment is performed to identify low coherence conditions, e.g., due to snow cover during winter acquisition.

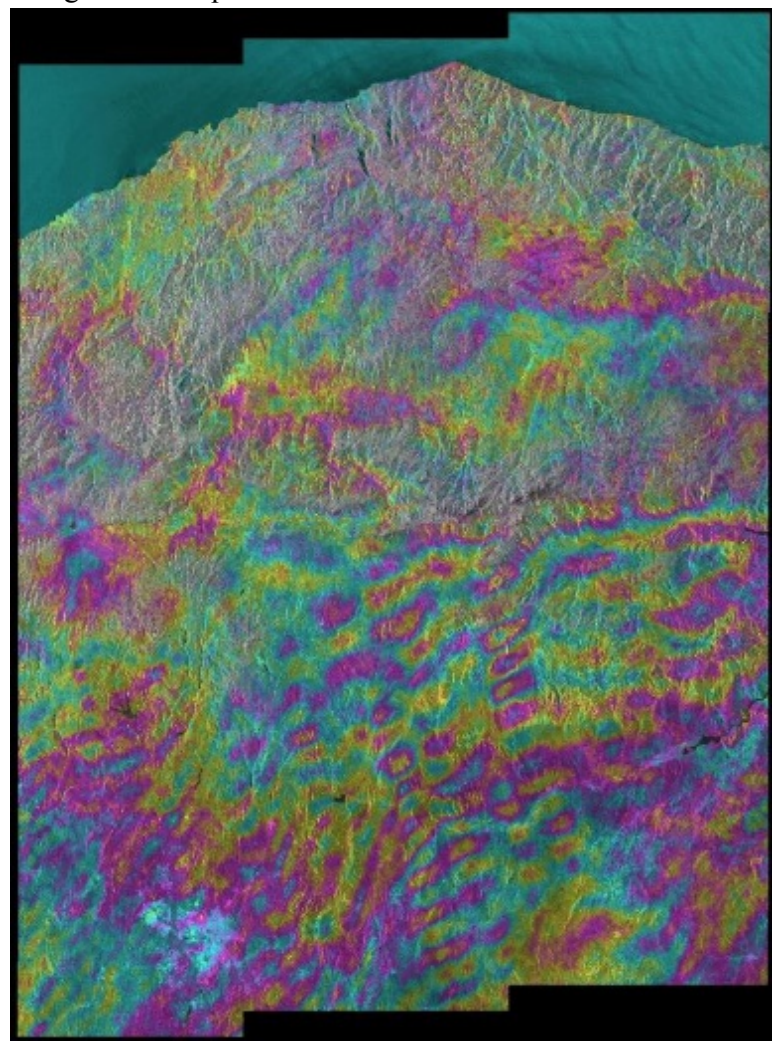

Figure 1. An interferogram processed using GAMMA on the CEMS/JASMIN system over Turkey. Using this facility allows COMET to undertake the large data processing requirements for the $\mathrm{LiCS}$ project. 
(a)

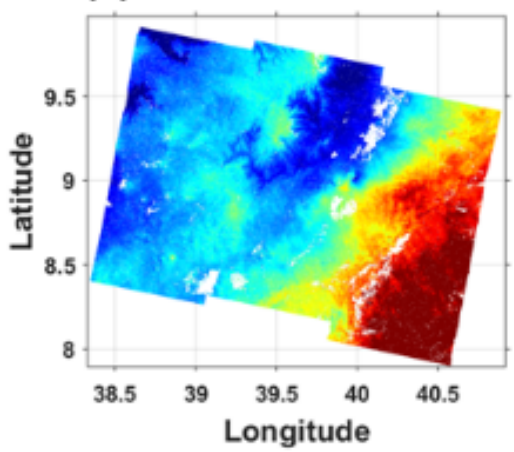

(b)

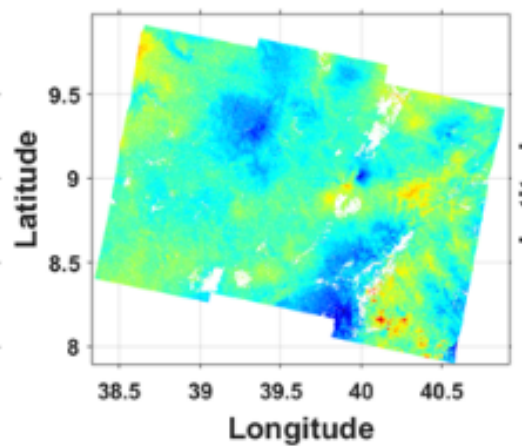

(c)

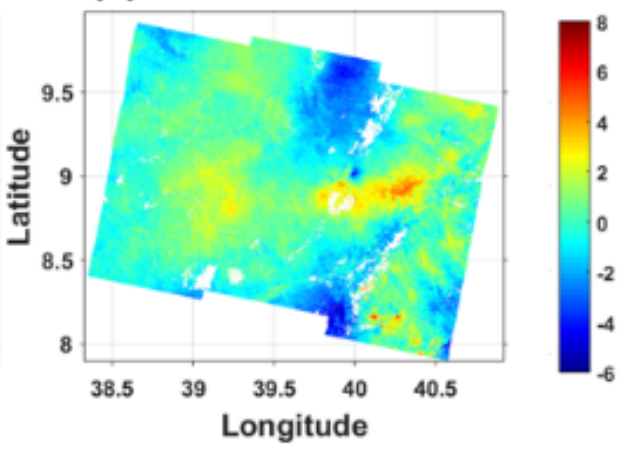

Figure 3. (a) Sentinel-1 interferogram (cm) over Ethiopia, during 28/03/2015-21/04/2015 and interferogram corrected using zenith total delays from (b) HRES-ECMWF and (c) ERA-Interim.

\section{HRES-ECMWF BASED ATMOSPHERIC CORRECTION MODEL}

The LiCS project team at Newcastle are assessing (i) the performance of numerical weather models (NWM) in describing the spatio-temporal variability of tropospheric water vapour, and (ii) the feasibility to use NWM products to reduce water vapour effects on InSAR measurements. More specifically we compare output from two global models: the operational high resolution European Centre for Medium range Weather Forecasting model (HRES-ECMWF) and the archived reanalysis products from ERA-Interim. HRES-ECMWF is run at a resolution of $\sim 16 \mathrm{~km}$ and is available near real-time (with a delay of 5-10 hours), whereas ERA-Interim has a resolution of $\sim 75 \mathrm{~km}$, is available with a few months delay and is currently the standard practice for NWM based InSAR corrections. The use of HRES ECMWF products, which are available near real-time, provides a unique contribution to COMET objectives of near-real time InSAR processing chain and quick responses to geohazards. Improved tropospheric delay estimates are found using HRES-ECMWF model output versus the coarser ERA-Interim products on a range of case studies. A systematic evaluation of HRES-ECMWF skills relative to ground and satellite based observations is ongoing, and the HRES-ECMWF based correction model is being incorporated into the COMET InSAR automatic processing chain.

\section{APPLICATIONS}

In the current phase, realistic scenarios have been targeted for key COMET research regions, for tectonics and volcanoes (Figure 4). For tectonic scenarios, we selected the North Anatolian Fault (Western Turkey). Here, we highlight in Figure 4, our frame processing strategy. Each Sentinel-1 TOPS scene product might contain a different set of bursts. We illustrate this in the right panel of Figure 4, showing the centre of bursts from different products (known as slices) as grey and black dots. Each frame polygon is defined to have a burst in common with the adjacent frame along the track. The common burst will be used to ensure spatial continuity of the large scale mapping products, e.g., the regional coherence and velocity maps.

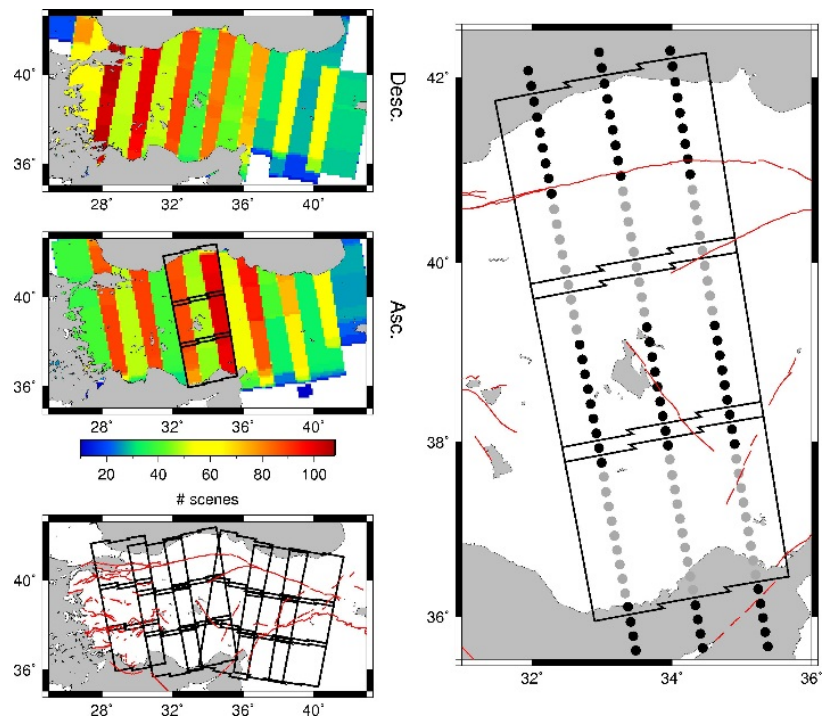

Figure 4. The North Anatolian Fault (Western Turkey) has been selected as a tectonic scenario.

For volcanic scenarios, we have responded to a number of volcanic unrest and eruptions during the past two years (20142016). For example, the 2014-2015 Fogo eruption in the Cape Verde Islands and the 2015 Calbuco sub-plinian eruption in Chile. We recently started to routinely produce deformation maps for selected volcano targets (e.g., Mt. Etna, Big Island of Hawaii, the Western Galápagos and Sakurajima volcano).

In Figure 5, we show Sentinel-1 interferograms over the Fogo Island. Here, we illustrate the regions of the burst overlap between adjacent bursts. Moreover, for geophysical applications the variable sensitivity to the along-track component of the surface displacement within each burst help to constrain better geophysical processes, such as the intrusion process associated to the 2014-2015 eruption at Fogo (González, et al., 2015). Results from the COMET processing chain would exploit routinely the redundancy of measurements in the burst overlap regions. This feature highlights the potential of Sentinel-1 TOPS interferometry for studies of Earth processes associated with natural hazards (e.g., volcanic and tectonic deformation). 


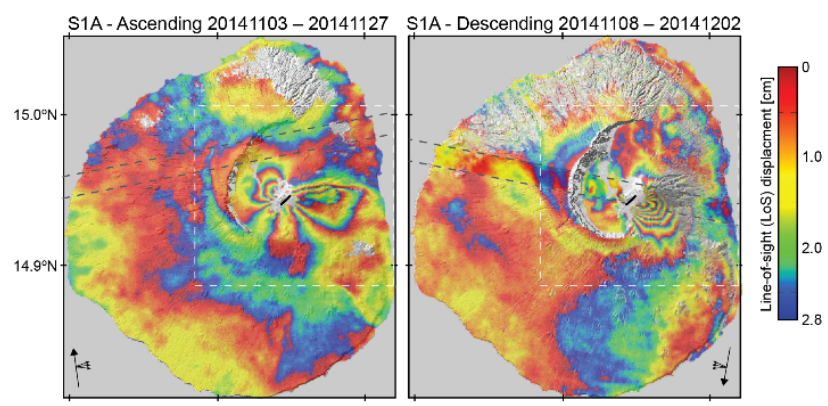

Figure 5. Sentinel-1 interferograms of the 2014-2015 eruption at Fogo Island. In the panels we show the location of the burst boundaries, denoting the burst overlap regions.

\section{CONCLUSIONS}

Currently, the COMET InSAR automated processing chain outputs products for predefined frames of the selected target areas (Figure 4). Updated surface displacement maps and time series products, are being used to generate large area linear velocity maps (e.g. Turkey). Assessment of the performance and the quality of the outputs generated by the COMET InSAR automated processing chain during the current phase will allow to scale-up the processing to larger regions in the near future.

The COMET InSAR automated system will provide a community-based facility to quickly inform about timedependent deformation events, and to analyse long-term small motions associated to geological processes. This geophysicscommunity facility will mark the beginning of an operational era of InSAR products to study active processes, everywhere, all the time.

\section{ACKNOWLEDGEMENTS}

This work was supported by the UK Natural Environmental Research Council (NERC) through the LICS project (ref. $\mathrm{NE} / \mathrm{K} 010794 / 1$ ) and the Centre for the Observation and Modelling of Earthquakes, Volcanoes and Tectonics (COMET, GA/13/M/031, http://comet.nerc.ac.uk). The Sentinel-1A interferograms presented are a derived work of Copernicus data, subject to the ESA use and distribution conditions.

\section{REFERENCES}

Elliott, JR, Searle, M, Jolivet, R et al., (2015). Himalayan megathrust geometry and relation to topography revealed by the Gorkha earthquake. Nature Geoscience. 9,174-180 (2016), doi:10.1038/ngeo2623.

Farr, T.G., Rosen, P.A., Caro, E., Crippen, R., Duren, R., Hensley, S., Kobrick, M., Paller, M., Rodriguez, E., Roth, L., Seal, D., Shaffer, S., Shimada, J., Umland, J., Werner, M., Oskin, M., Burbank, D., Alsdorf, D., 2007, The Shuttle Radar Topography Mission. Reviews of Geophysics 45: RG2004 doi:10.1029/2005RG000183.

González, P. J., M. Bagnardi, A. J. Hooper, Y. Larsen, P. Marinkovic, S. V. Samsonov, and T. J. Wright (2015), The 20142015 eruption of Fogo volcano: Geodetic modeling of Sentinel1 TOPS interferometry, Geophys. Res. Lett., 42, 9239-9246, doi:10.1002/2015GL066003. 